MATEC Web of Conferences 23, 01068 (2015)

DOI: $10.1051 /$ matecconf/ 20152301068

(C) Owned by the authors, published by EDP Sciences, 2015

\title{
HEAT AND MASS TRANSFER AT HOT SURFACE IGNITION OF COAL PARTICLE
}

Dmitrii O. Glushkova ${ }^{\mathrm{a}}$, Andrey. G. Kosintsev, Nikita E. Shlegel, Ksenia Yu. Vershinina

National Research Tomsk Polytechnic University, 634050 Tomsk, Russia

\begin{abstract}
This paper describes the experimental investigations of the characteristics of heat and mass transfer during the conductive heating of a coal particle. We have established the boundary conditions of combustion initiation, and the conditions of thermal decomposition and solid fuel particles decay, characterized by the temperature of a heat source, and the duration of the respective stages.
\end{abstract}

\section{INTRODUCTION}

Fast physical and chemical processes [1-4] during heating, ignition and combustion of coal particles are of great interest due to the wide range of their applications. Currently, most boilers operate with natural solid fuels (coal) [5]. Coal should be preliminarily prepared (grinding, drying) in a fuel preparation system for its futher combustion in the furnace [6]. A particle size varies from a few centimeters to several tens of micrometers. Fuel preparation is inherently associated with coal heating to provide acceptable humidity level. It should be noted that coal particles can interract with heated surfaces at the different stages of technological process. In order to prevent accidents (unregulated solid fuel ignition), information is required about the temperature limits and characteristic times of induction progression.

Most publications with the results of theoretical and experimental studies (for example, [7, 8]) are associated with high-temperature ignition. The analysis of the theory of solid fuels combustion [9] shows that heat transfer mechanisms and conditions are different when heat source temperature vary from $500 \mathrm{~K}$ to $2000 \mathrm{~K}$. Therefore, it is advisable to perform the experimental studies of the integral characteristics of coal particle ignition at conductive heating.

The aim of this paper is the experimental study of heat and mass transfer at the hot surface ignition of single hot particles.

\section{EXPERIMENTAL SETUP AND PROCEDURE}

Fig. 1 shows a setup for the experimental investigation of coal particle ignition during conductive heating.

The operation principle of the setup (Fig. 1) is as follows. An induction heating system 1 and 2 heats a metal plate (steel). The plate is placed inside an induction coil (inductor). As a result of the alternating magnetic field, the meatal plate is heated due to resistance and eddy currents. A chiller 3 is intended for the water cooling of generator power elements and the induction coil made from hollow

${ }^{\mathrm{a} C}$ Corresponding author: dmitriyog@tpu.ru 
copper tubing. Measurement and maintaining the desired temperature of the steel plate surface is carried out using an infrared pyrometer 4 and a controller 5 with the function of actuators discrete control. Deadband for maintaining the temperature of the metal plate at a predetermined level is $\pm 5 \%$. High-speed video camera 6 records fast processes in the vicinity of the plate surface during the induction period. Video saving and processing is carried out in computer 7 using specialized software.

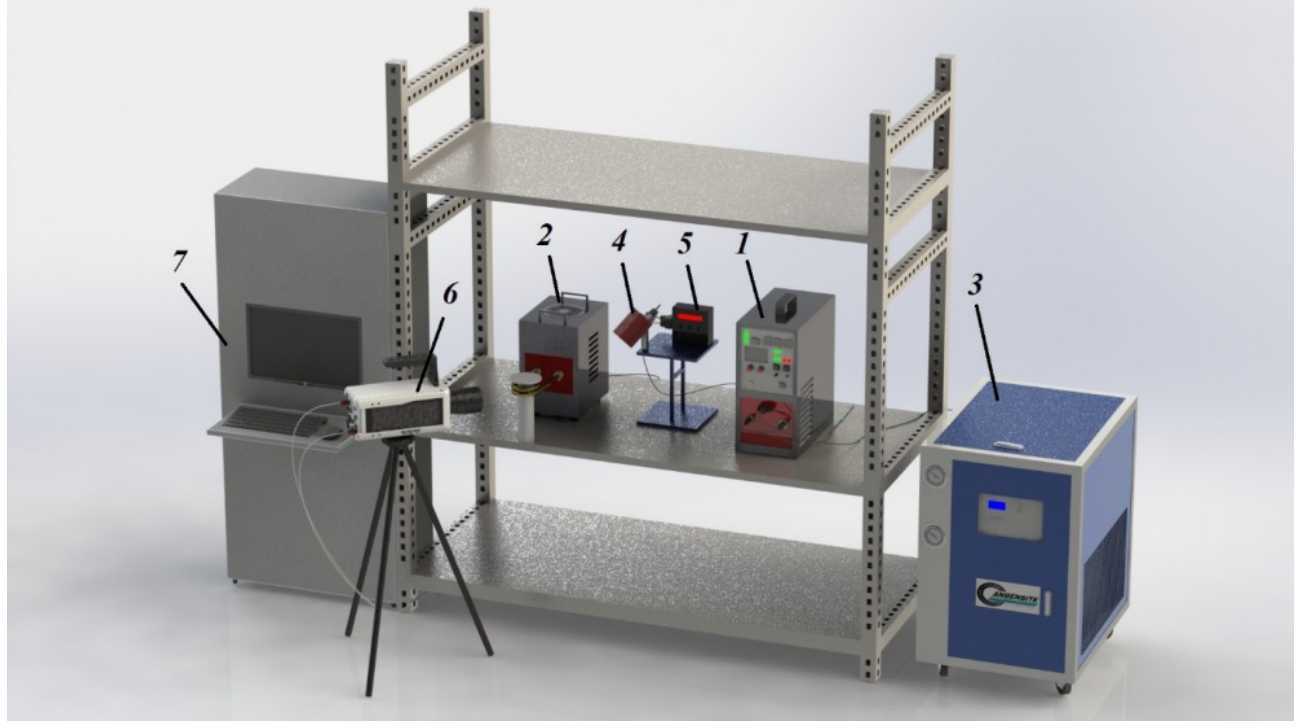

Figure 1. Scheme of an experimental setup: 1 - generator; 2 - induction heater; 3 - chiller; 4 - infrared pyrometer; 5 - temperature controller; 6 - high-speed video camera; 7 - computer.

Coal particles larger than $1 \mathrm{~mm}$ are in the form of irregular polyhedra; the sizes of polyhedron faces are very different. Since the conditions of the conductive heating of particles depend essentially on the contact area with a heat source, there may be different versions of heat and mass transfer during the ignition of the same particle at different positions on the heating surface. When planning the experiment, we chose polyhedron with similar dimensions in three spatial directions from a large variety of the possible shapes of particles.

An induction heater 2 heated the disc-shaped metal plate to a predetermined temperature. After the temperature had been stabilized, a coal particle was placed on the heating surface. Video recording started at the moment of contact between the particle and the plate. Ignition delay time $t_{d}$ was determined from video recordings and was counted until the flame.

\section{Results and discussion}

For experimental studies we selected coal particles as samples with the sizes of $4 \mathrm{~mm}, 5 \mathrm{~mm}$ and 6 $\mathrm{mm}$ (Fig. 2). We carried out a series of experiments for each characteristic size at a fixed temperature of the heat source. Each series of experiments consisted of not less than six experiments. We determined the basic characteristic of the process - ignition delay time $t_{d}$. We performed subsequent analysis to average the experimental data.

Processing results obtained using the setup (Fig. 1) established dependencies (Fig. 3) of the ignition delay time of coal particles with various sizes from the temperature of the heating surface. 


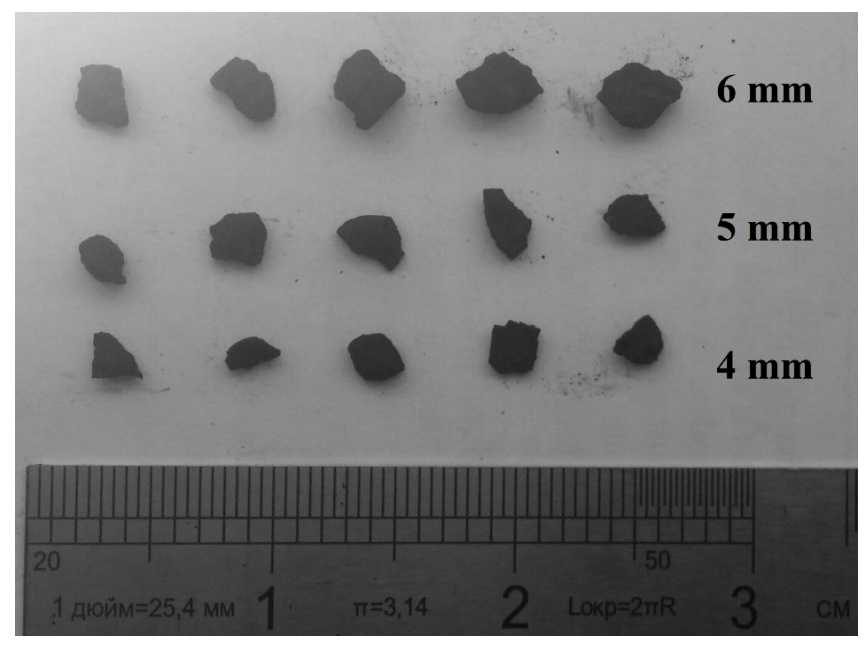

Figure 2. Coal particles with the sizes of $4 \mathrm{~mm}, 5 \mathrm{~mm}, 6 \mathrm{~mm}$.

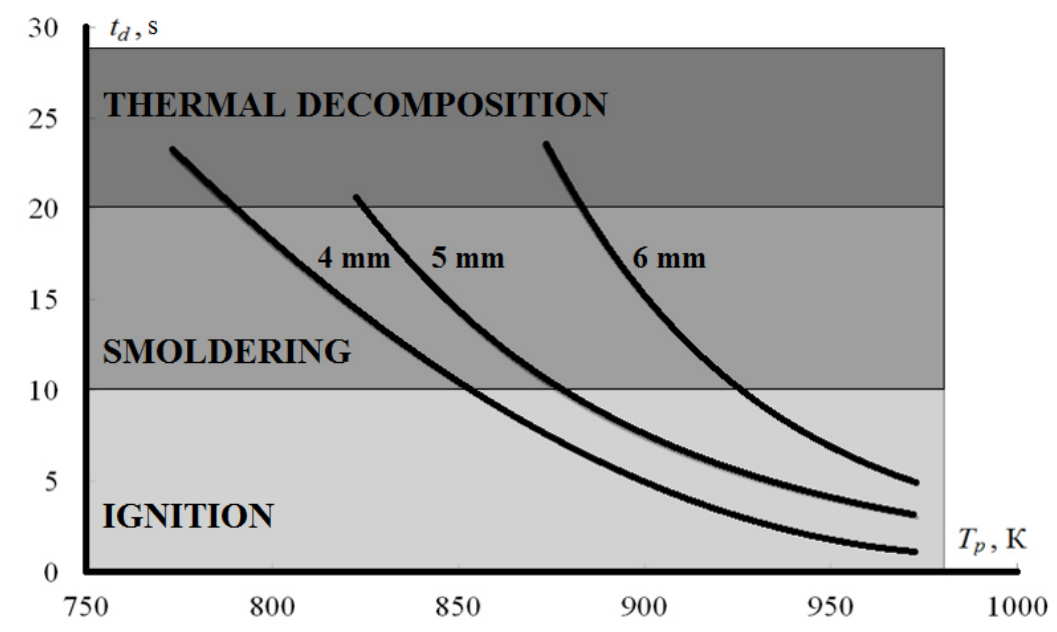

Figure 3. Dependencies of the ignition delay time of coal particles from the temperature of the heating surface.

Experimental data (Fig. 3) shows that ignition delay time increases with the increasing of the particle size at a fixed temperature of the heat source. Also, there is a change in the limit (minimum) temperatures of the plate, necessary and sufficient to initiate solid fuels combustion. It was found that ignition occurs for particles with a size of $4 \mathrm{~mm}$ at $850 \mathrm{~K}, 5 \mathrm{~mm}$ at $880 \mathrm{~K}, 6 \mathrm{~mm}$ at $930 \mathrm{~K}$. This result is explained as follows. The uniform heating of particles with smaler sizes lasts for a short time period (in comparison with larger particle size). In this process, the concentration of combustible gas mixture components (volatile and oxidizer) is high in the vicinity of the particle during the induction period due to the high intensity of coal thermal decomposition.

The ignition of volatiles occurs as a result of their additional heating from the hot plate. The oxidation occurs in the gas medium and is accompanied by energy release, that leads to the heating of coke residue and its subsequent ignition (Fig. 4). 

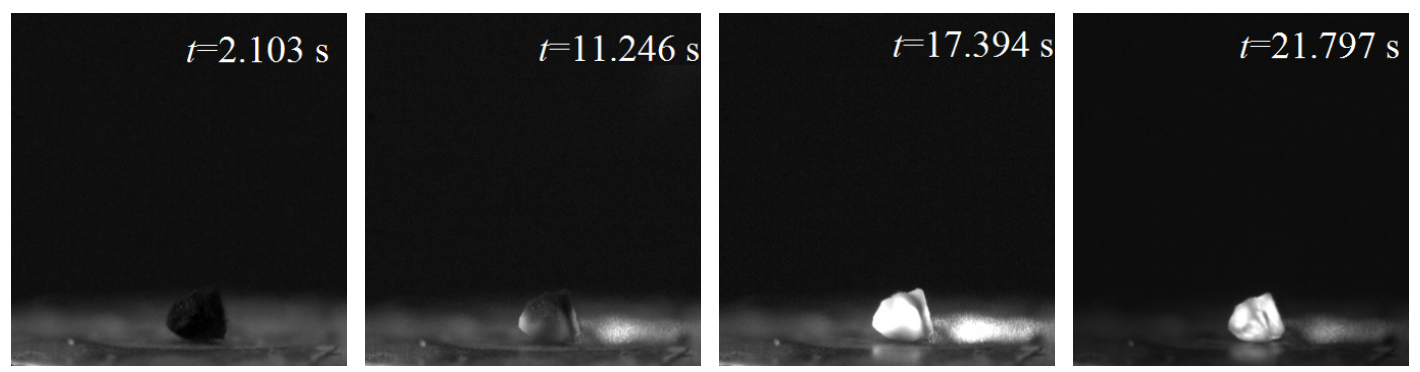

Figure 4. Stages of coal particle ignition.

\section{Conclusion}

Experimental studies established the boundary conditions of the ignition of coal particles with different sizes: $4 \mathrm{~mm}-850 \mathrm{~K}, 5 \mathrm{~mm}-880 \mathrm{~K}, 6 \mathrm{~mm}-930 \mathrm{~K}$. Particles ignition does not occure at the lower temperatures of the ignition source. As a result of their heating, thermal decomposition or decay of fuel particles occurs.

The study was financially supported by Russian Foundation for Basic Research (project No. 14-0331304 mol_a). The experimental setup was developed with the financial support of the program to improve the competitiveness of Tomsk Polytechnic University (project VIU_ENIN_2014).

\section{References}

1. C. Zou, L. Cai, C. Zheng, Int. J. Heat Mass Transfer 73, 207 (2014).

2. D.O. Glushkov, G.V. Kuznetsov, P.A. Strizhak, Russ. J. Phys. Chem. B. 9, 242 (2015).

3. H. Fujitsuka, R. Ashida, M. Kawase, K. Miura, Energy Fuels 28, 2402 (2014).

4. D.O. Glushkov, P.A. Strizhak, O.V. Vysokomornaya, Therm. Sci. 19, 285 (2015).

5. A. Askarova, S. Bolegenova, V. Maximov, M. Beketayeva, P. Safarik, J. Therm. Sci. 24, 275 (2015).

6. F. Fu, M. Kong, C. Xu, C. Liang, S. Wang, Adv. Mech. Eng. 2013, 656194 (2013).

7. S.-W. Du, C.-P. Yeh, W.-H. Chen, C.-H. Tsai, J.A. Lucas, Fuel 143, 98 (2015).

8. C. Gonzalo-Tirado, S. Jiménez, R. Johansson, J. Ballester, Combust. Flame 161, 1085 (2014).

9. D.A. Frank-Kamenetsky, Diffusion and Heat Transfer in Chemical Kinetics (New York, Plenum, 1969). 